\title{
Case Report: The First Direct Evidence of Gnathostoma spinigerum Migration through
}

\section{Human Lung}

\author{
Chaisith Sivakorn, ${ }^{1}$ Kingpeth Promthong, ${ }^{2}$ Paron Dekumyoy, ${ }^{3}$ Parnpen Viriyavejakul, ${ }^{4}$ Sumate Ampawong, ${ }^{4}$ Wallop Pakdee, ${ }^{3}$ \\ Kittipong Chaisiri, ${ }^{3}$ and Dorn Watthanakulpanich ${ }^{3 *}$ \\ ${ }^{1}$ Department of Clinical Tropical Medicine, Faculty of Tropical Medicine, Mahidol University, Bangkok, Thailand; ${ }^{2}$ Department of Pulmonary and \\ Critical Care Medicine, Maharaj Nakhon Si Thammarat Hospital, Nakhon Si Thammarat, Thailand; ${ }^{3}$ Department of Helminthology, Faculty of \\ Tropical Medicine, Mahidol University, Bangkok, Thailand; ${ }^{4}$ Department of Tropical Pathology, Faculty of Tropical Medicine, Mahidol University, \\ Bangkok, Thailand
}

\begin{abstract}
Gnathostomiasis is a helminthic infection caused by the third-stage larvae of nematodes of the genus Gnathostoma. The life cycle in humans starts with an enteric phase, with the worm perforating the gastric or intestinal mucosa to reach the peritoneal cavity and migrating through the human body. Subsequent penetration through the diaphragm may produce pleuropulmonary symptoms. We herein present a previously healthy 56-year-old Thai man from Southern Thailand who was an ex-smoker presented with chronic dry cough progressing to hemoptysis after consuming grilled swamp eels and freshwater fish. Chest computed tomography showed consolidation at the lingular segment, and the differential diagnosis was primary lung cancer and pulmonary tuberculosis. The lung tissue biopsied during bronchoscopy displayed segments of organisms with the phenotypic characteristics of Gnathostoma spp., and abundant eosinophils were seen in the alveolar tissue. Gnathostoma spinigerum infection was confirmed by a Western blot assay for G. spinigerum-specific 24-kDa reactive band. The patient received albendazole, and a follow-up chest radiograph revealed improvement in the consolidation in the lung and reduction in hemoptysis. We report the first direct evidence including pathology and immunohistochemistry of Gnathostoma invasion via the human lung, with clinical and radiographic presentations mimicking either malignancy or chronic infection.
\end{abstract}

\section{INTRODUCTION}

Gnathostomiasis is prevalent in Southeast Asian countries, ${ }^{1,2}$ East Asia, and Latin America, ${ }^{3}$ and it is sporadically found in other parts of the world mainly because of a patient visiting an endemic area. ${ }^{4}$ Gnathostoma larvae migrate to the skin, ${ }^{5,6}$ gastrointestinal system, genitourinary system, auditory system, central nervous system, and the eyes, and parasitosis of the central nervous system may cause life-threatening complications. ${ }^{7-9}$ Definite diagnosis is made by identification of the parasite taken from the patient or through histopathological section of the suspected lesion. The clinical presentations of gnathostomiasis in the human lung were previously described as transient pulmonary lesions with a marked increase in eosinophils in both blood and sputum (Loeffler's syndrome) ${ }^{7}$ and spontaneous pneumothorax. ${ }^{10}$ To the best of our knowledge, no gnathostomiasis study in humans has demonstrated the parasite in the lung tissue, as all previous diagnoses for pulmonary gnathostomiasis depended on evidence of migratory swelling or cutaneous creeping eruption along with transient abnormal chest radiograph. ${ }^{7}$ In the present case, human pulmonary gnathostomiasis was diagnosed by a lung tissue biopsy taken during bronchoscopy supported with confirmatory organismspecific Western blotting, which is the first direct demonstration of Gnathostoma larval migration to the lung in humans.

\section{CASE REPORT}

A previously healthy 56-year-old Thai man presented with dry cough for 3 months without fever or dyspnea. He was a 20-packyear ex-smoker, had no current medication, and lived in Nakhon

\footnotetext{
* Address correspondence to Dorn Watthanakulpanich, Department of Helminthology, Faculty of Tropical Medicine, Mahidol University, 420/6 Ratchawithi Rd., Ratchathewi, Bangkok 10400, Bangkok,
} Thailand. E-mail: dorn.wat@mahidol.edu
Si Thammarat Province in Southern Thailand. He had tried antitussive agents without improvement. He had a history of regular consumption of grilled swamp eels and freshwater fish, especially snakehead fish and catfish. He denied either consuming raw or undercooked meats or drinking unclean water. He had a pet cat and a vegetable plot fertilized with organic kitchen waste, where he walked barefooted. He had no known contact with tuberculosis and reported no anorexia or weight loss. He did not notice any cutaneous migratory swelling or creeping eruption. He denied any travel outside his province. Two months after the onset of his cough, he developed hemoptysis. He took a 7-day course of amoxicillin, but his symptoms did not improve.

On physical examination at presentation, he had a body temperature of $36.8^{\circ} \mathrm{C}$, a pulse rate of 86 beats/minutes, a respiratory rate of 14 breaths/minutes, a blood pressure of $118 / 60 \mathrm{mmHg}$, and an oxygen saturation of $98 \%$ while breathing room air. He did not have cyanosis, clubbing, pursed lip expiration, accessory respiratory muscle usage, and nasal flaring. Auscultation of the thorax revealed bronchial breath sound at the left anterior thorax below the nipple and coarse crepitation of the left lung, but no wheezing. The initial chest radiograph showed alveolar opacity silhouette with the left heart border (Figure 1A), whereas the chest computed tomography (CT) showed pan lobular emphysema of the upper lobe and consolidation with central necrosis of the lingular segment (Figure 1C-F). The initial complete blood count showed mild eosinophilia (absolute count 400 cells $/ \mu \mathrm{L}$ ) without other abnormalities. In sputum Gram stain, acid-fast bacilli were negative and sputum cultures for both bacteria and Mycobacterium reported later were negative. The differential diagnosis was primary lung cancer and chronic infection with Mycobacterium tuberculosis because of his history of being an ex-heavy smoker and CT findings. Bronchoscopy with tissue biopsy was performed, and empirical antibiotic (amoxicillin-clavulanic acid $875 \mathrm{mg} / 125 \mathrm{mg}$ two tablets per 


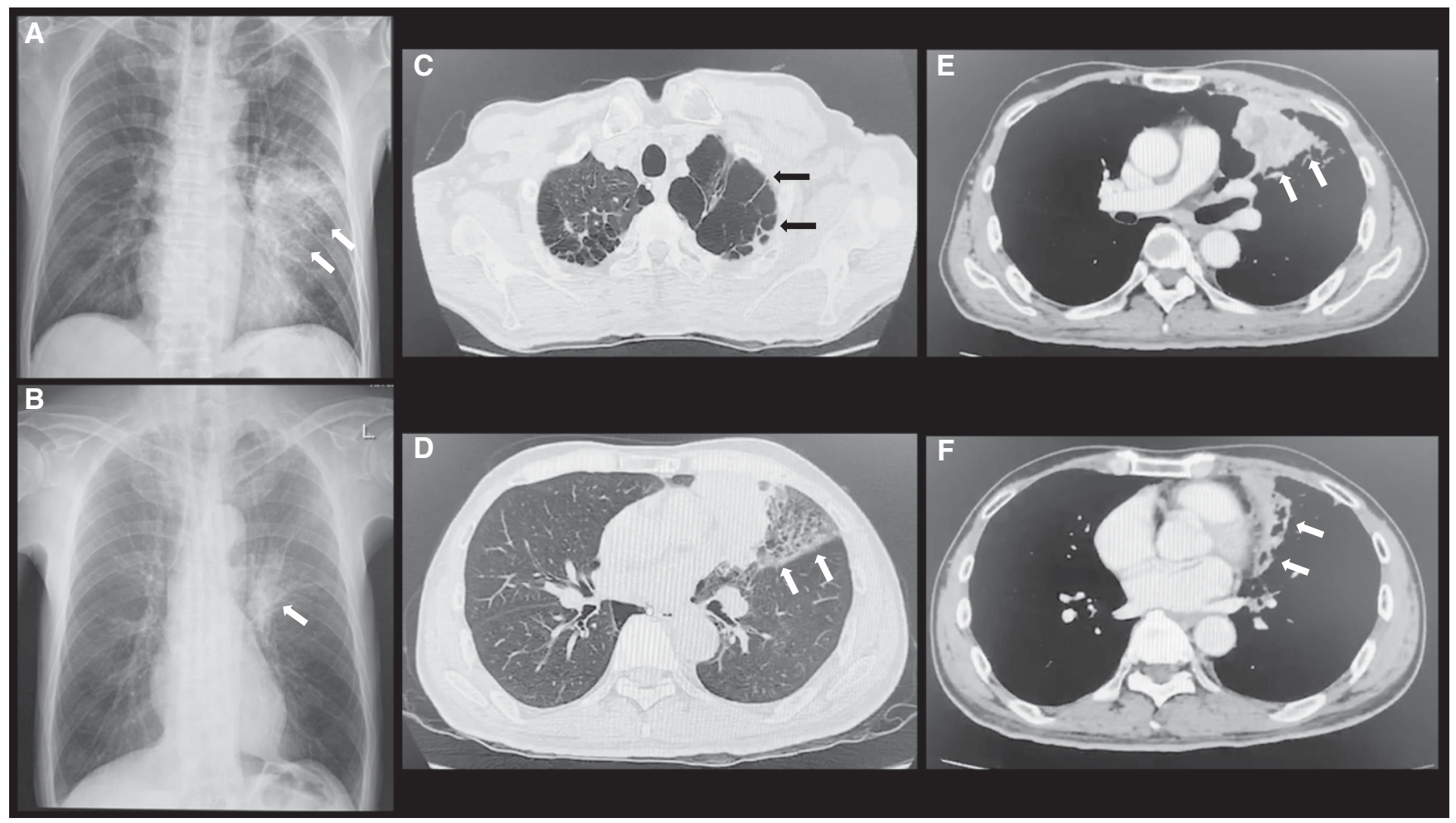

FIGURE 1. Chest radiograph and computed tomography (CT) of the patient were demonstrated. (A): The initial chest radiograph showed alveolar opacity silhouette with the left heart border (arrows). (B): The 1-month follow-up chest radiograph after bronchoscope and albendazole treatment showed improvement of alveolar opacity (arrow). (C-F): CT chest with contrast shows panlobular emphysema of both upper lobes (arrows) (C) and consolidation with central necrosis of the lingular segment (arrows) (D-F).

day for 14 days) was given. The hematoxylin and eosin (H\&E) stain of the biopsied lung tissue revealed segments of parasites with lateral cuticular spines, undulating cuticles, and coelomic muscles, prompting diagnosis of pulmonary gnathostomiasis (Figure 2). Fragments of the alveolar tissue, clusters of inflammatory cells, particularly lymphocytes, and abundant eosinophils were also observed. In-house confirmatory Western blot assay was positive for Gnathostomiasis spinigerum, specifically $24-\mathrm{kD}$ a reactive band (Figure 3$)^{2}$ An immunohistochemical study was performed to examine the level of anti-inflammatory and pro-inflammatory cytokines in the peribronchiolar and alveolar tissues from the biopsied lung compared with the normal lung. $\mathrm{H}$-score (0-300) was used to determine the expression of those cytokines by the multiplication of intensity score (0-3; 0 : absent and 1-3: weak, moderate, and strong reactivities, respectively) and the percentage area of expression/field (0-100\%). Digitalized image analysis program (Image J, $\mathrm{NIH}$ ) was used to examine the area of expression. ${ }^{11-13}$ In brief, 10 random fields of color images from each group were acquired and converted to gray scale. Adjusted images were transformed by the threshold mode to locate the area of positive reaction and estimated by the number of black pixels. Thus, the area fraction of positive reaction was determined as the percentage of black pixels/area. The results indicated that there was greater expression of the transforming growth factor (TGF)- $\beta$ in the peribronchial area than interleukin (IL)-4, IL-10, and tumor necrotic factor (TNF)- $\alpha$ (Figure 4). In addition, the alveolar tissue from patients also exhibited the upregulation of TGF- $\beta$ when compared with the normal tissue. However, the levels of IL-4, IL-10, and TNF- $\alpha$ were lower in the patient's lung than those in the normal lung (Figure 5).

The patient was informed the diagnosis of pulmonary gnathostomiasis and ensured the curative treatment because of accidental removal of parasite by tissue biopsy under bronchoscopy. In the follow-up, the chief complains of dry cough and hemoptysis symptoms disappeared, and the physical examination revealed normal finding. None of the bronchial breath sound was detected from chest auscultation together with improvement of alveolar opacity from chest radiograph (Figure 1). Complete blood count showed a decrease in absolute eosinophil count (54 cells $/ \mu \mathrm{L})$. A routine stool examination performed to identify any other parasites was negative.

\section{DISCUSSION}

Humans are an accidental host of Gnathostoma spp. Foodborne zoonosis occurs by ingesting uncooked meats of secondary intermediate hosts in its zoonotic life cycle, mainly freshwater fish or paratenic animals, such as fish-eating reptiles and birds. ${ }^{14,15}$ Our patient had a risk of infection because of habits of consuming grilled swamp eels and some freshwater fish that may have been undercooked. He consumed snakehead fish and catfish, which were proven as important intermediate/paratenic hosts. ${ }^{16,17}$ Also, 48 animal species were reported as reservoir hosts for transmission of infective Gnathostoma larvae to humans in Thailand. ${ }^{18}$ The larval and immature stages of Gnathostoma spp. are hardy and may tolerate the heat if located deep in the flesh or internal organs of improperly grilled fish. 


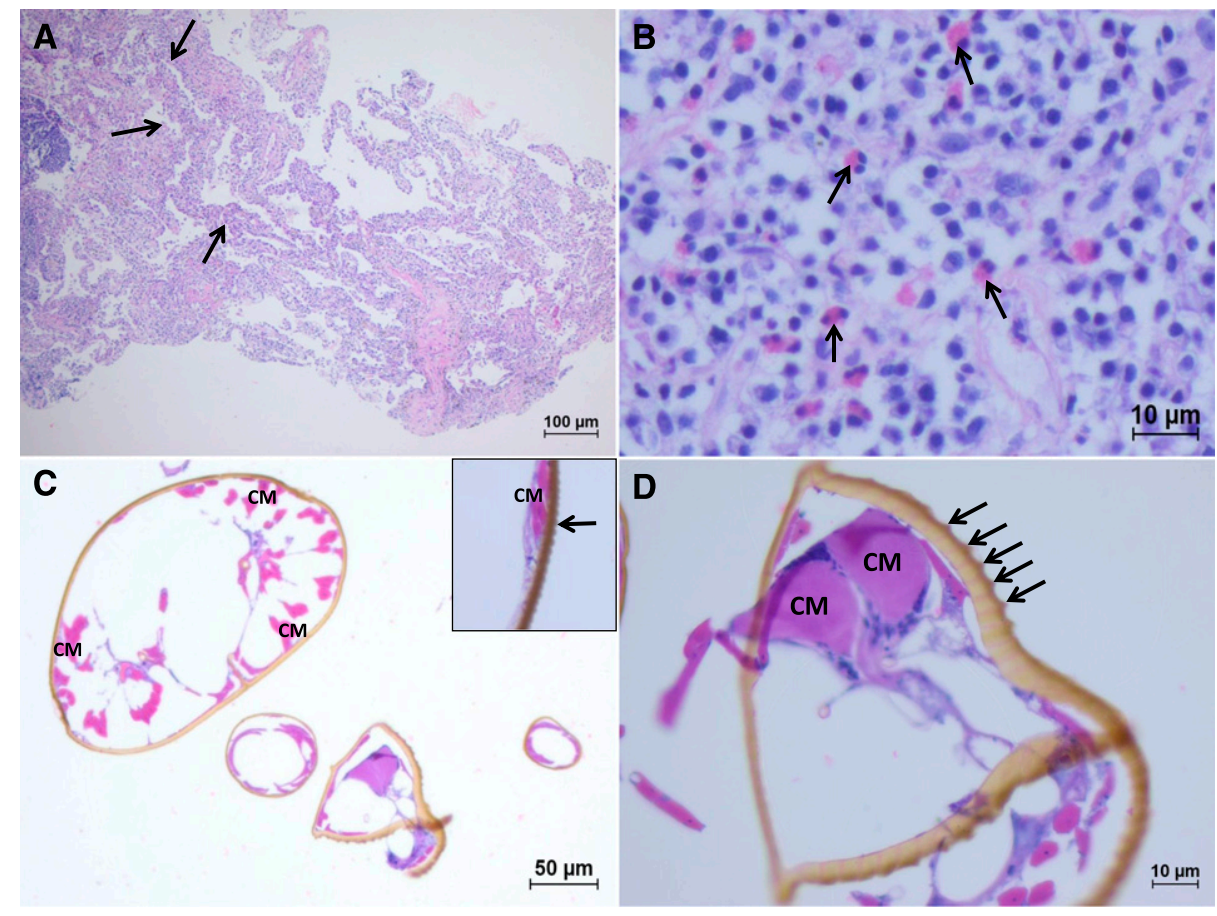

FIGURE 2. Details of the biopsied lung were displayed. $\mathbf{A}$ and $\mathbf{B}$ : Biopsy from the lung tissue shows thickening of the alveolar wall (A, hematoxylin and eosin [H\&E] stain 50x) and pronounced eosinophilic infiltration (B, H\&E stain 400x). (C and D): Fragments of the cross-sectioned Gnathostoma worm were illustrated (C, H\&E stain 100x), with higher magnification of the undulating cuticles (inset) from the larger parasite fragment. Another parasite fragment displayed lateral pointed cuticular spines (D, H\&E stain $400 \times)$. CM = coelomic muscles. This figure appears in color at www.ajtmh.org.

Our patient had Gnathostoma larvae in the biopsied lung tissue, which was rare direct evidence of migration to the lung. In most infected cases, especially with pulmonary involvement, the larvae of G. spinigerum were not detected..$^{7,19,20}$ Therefore,
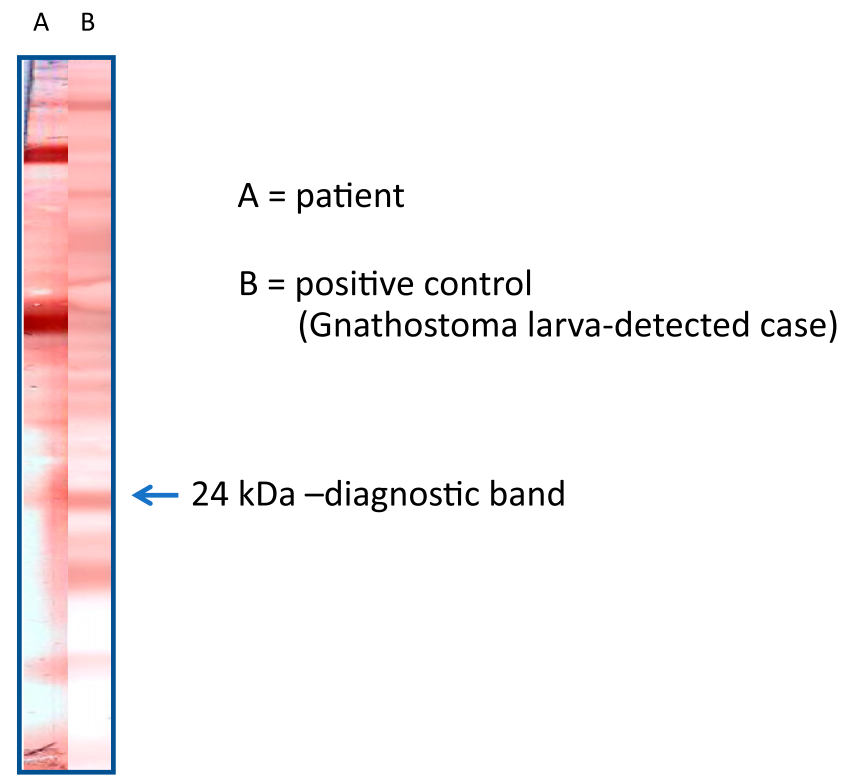

FIGURE 3. Specifically reactive band by Western blot analysis was diagnosed as gnathostomiasis. (A): Patient's serum and (B): positive control (patient's serum with Gnathostoma larva detected); the diagnostic reactive band of gnathostomiasis was indicated by arrow. This figure appears in color at www.ajtmh.org. a presumptive diagnosis is usually made based on the clinical manifestations of a migratory swelling and a history of consuming of freshwater fish or other intermediate or paratenic hosts in an endemic area. Pulmonary gnathostomiasis is often preceded by episodes of migratory swelling over a period of years ${ }^{21-24}$ together with chest symptoms of cough, ${ }^{19,23-25}$ varying from paroxysmal dry to violent cough, ${ }^{25}$ pleuritic pain, ${ }^{10,23,24}$ dyspnea, ${ }^{22}$ hemoptysis, ${ }^{20,22}$ lobar consolidation or collapse, ${ }^{21}$ pleural effusions, ${ }^{21-23}$ pneumothorax, ${ }^{10}$ and hydropneumothorax. ${ }^{23}$ Thoracentesis was performed in a case with pleural effusion, demonstrating exudative pleural fluid with abundant eosinophils. ${ }^{10,23}$ In all previously reported cases, there was no direct evidence of pulmonary invasion except for indefinite chest symptoms and/or the expectoration of worms. In several cases from Thailand, the larval worm migrated to the upper respiratory tract or even to the pharynx; then, it was ultimately expectorated, and chest symptoms were resolved. ${ }^{24,26}$ In other endemic Asian countries, such as China and Japan, pulmonary gnathostomiasis was reported sporadically and was diagnosed by pleuritic pain and nodular lesion on chest CT and supported by rising antibodies in patient's serum detected with a serological test. ${ }^{27,28}$

In the present study, we found the G. spinigerum larval stage in the human lung by the tissue biopsy technique, and the Western blot assay confirmed the diagnosis by organismspecific 24-kDa reactive band with the antibody against G. spinigerum. ${ }^{2}$ As clinical manifestations and radiographic findings of pulmonary gnathostomiasis are not specific and scanty, morphological identification of Gnathostoma larval detection including histopathology, immunohistochemistry, 

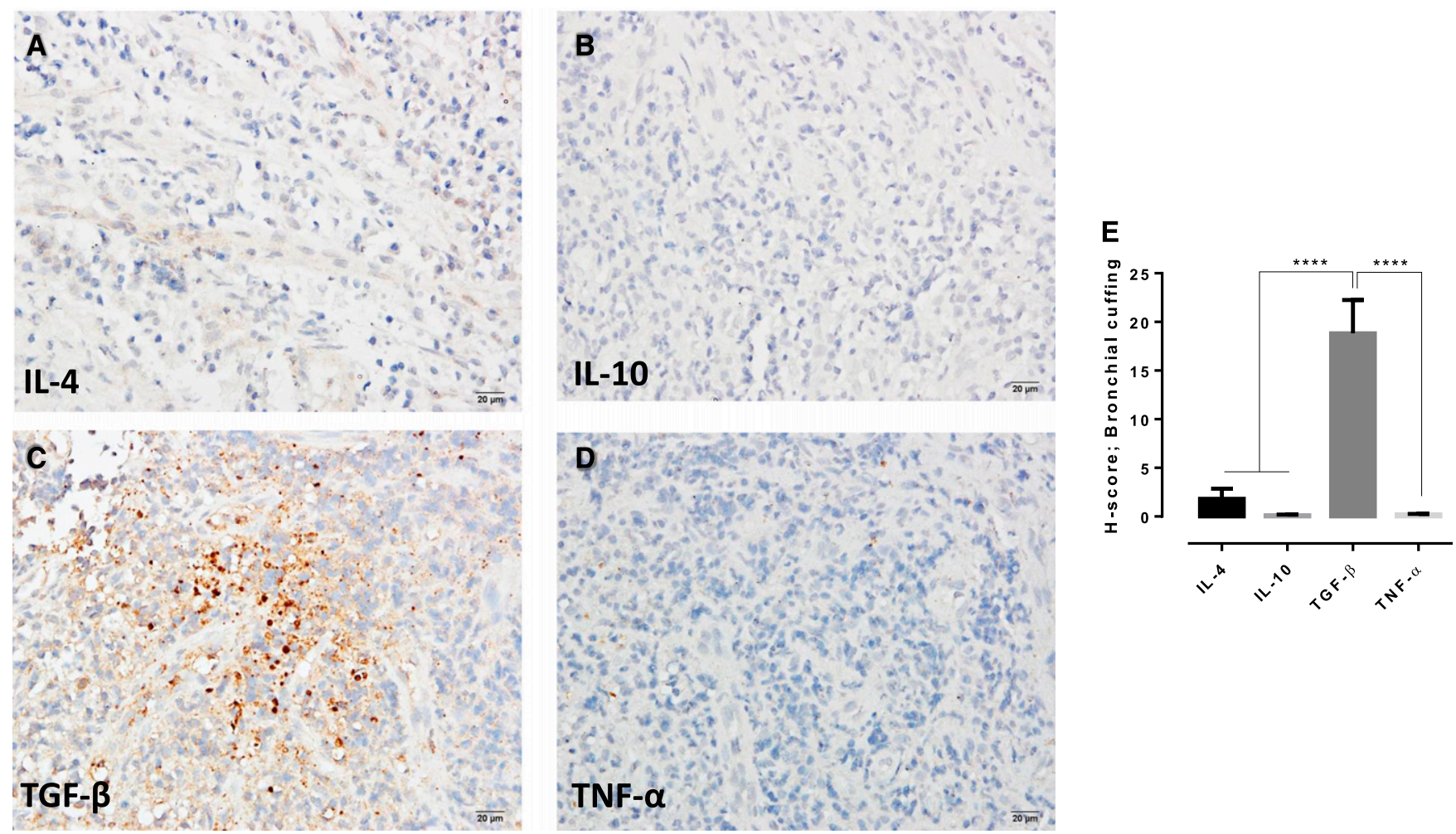

FIGURE 4. Expressions of anti-inflammatory and pro-inflammatory cytokines were demonstrated. (A-E): interleukin (IL)-4, IL-10, transforming growth factor- $\beta$, and tumor necrotic factor- $\alpha(\mathbf{A}-\mathbf{D})$ scoring with $H$-score $(\mathbf{E})$ in the peribronchial tissue from lung biopsy by immunohistochemical staining. This figure appears in color at www.ajtmh.org.

and confirmatory Western blotting is useful for definite diagnosis. However, obtaining the larval worm from a patient via the biopsied lung tissue is difficult because of its very small size and migrations. Moreover, the biopsy technique may reduce the specificity of morphological diagnosis because of distortions in the organism or only a small part of the larval worm being extracted from the lung tissue. However, some characteristics of the worm can be observed, allowing for identification. These included several characteristic features of the body shape, the number of rows of hooks at the cephalic end, the number of hooks in each row, the character of the spines which cover the body, and the extent to which the body was covered by spines, which vary according to species. ${ }^{29,30}$ In the present case, histopathological findings of the fragmented parasite with features of lateral cuticular spines, undulating cuticles, coelomic muscles, and eosinophilic response were highly suggestive of Gnathostoma spp.

Host inflammatory responses to the parasitic diseases have been discussed for many decades. Acute inflammation with eosinophil and neutrophil infiltrations or chronic granulomatous inflammation was mainly observed depending on disease progression. In gnathostomiasis, host cellular immune responses with the production of cytokines, for example, IL-4, IL-5, IL-10, and IL-13, were described in promoting granulomatous formation. ${ }^{31}$ However, encyst formation has never been reported in the human gnathostomiasis. Interleukin-5 plays a crucial role in growth, differentiation, and activation of eosinophils ${ }^{32}$ in relation to helminth-induced eosinophilia and eosinophilic inflammation. In the present study, high levels of circulating and tissue eosinophilia were observed which would be a result of upregulation of IL-5. Further studies on IL-5 levels are required in gnathostomiasis. Moreover, helminthic secretions induce T-cell Foxp3 expression through the TGF- $\beta$ pathway, and this regulates the immune response to create an environment for the parasite to persist. ${ }^{33}$ Recently, the level of Fc gamma receptor I, a high-affinity receptor for initial phagocytic signaling, was inhibited by $G$. spinigerum excretory-secretory products, ${ }^{34}$ which was similar to the effect from TGF- $\beta$ production. ${ }^{35}$ The immunohistochemical study of our patient's biopsied lung tissue showed upregulation of TGF$\beta$ compared with IL-4, IL-10, and TNF- $\alpha$. This evidence in the infected human lung tissue supports those of the previous in vitro and animal model studies for the role of TGF- $\beta$ in retarding the host's innate immune response.

In conclusion, we report the first direct evidence of Gnathostoma migration to the human lung with clinical presentation mimicking either malignancy or chronic infection. Confirmatory Western blot assay, histopathology, and additional immunohistochemistry are useful for definite diagnosis.

Received April 2, 2020. Accepted for publication May 26, 2020.

Published online June 22, 2020.

Acknowledgments: We would like to express gratitude to the patient and the staff of the Faculty of Tropical Diseases, Bangkok, Thailand, and the staff of Department of Pulmonary and Critical Care Medicine, Maharaj Nakhon Si Thammarat Hospital, Thailand.

Financial support: The publication of this work was granted by the Faculty of Tropical Medicine, Mahidol University.

Authors' addresses: Chaisith Sivakorn, Department of Clinical Tropical Medicine, Faculty of Tropical Medicine, Mahidol University, Bangkok, Thailand, E-mail: chaisith.siv@mahidol.edu. Kingpeth 


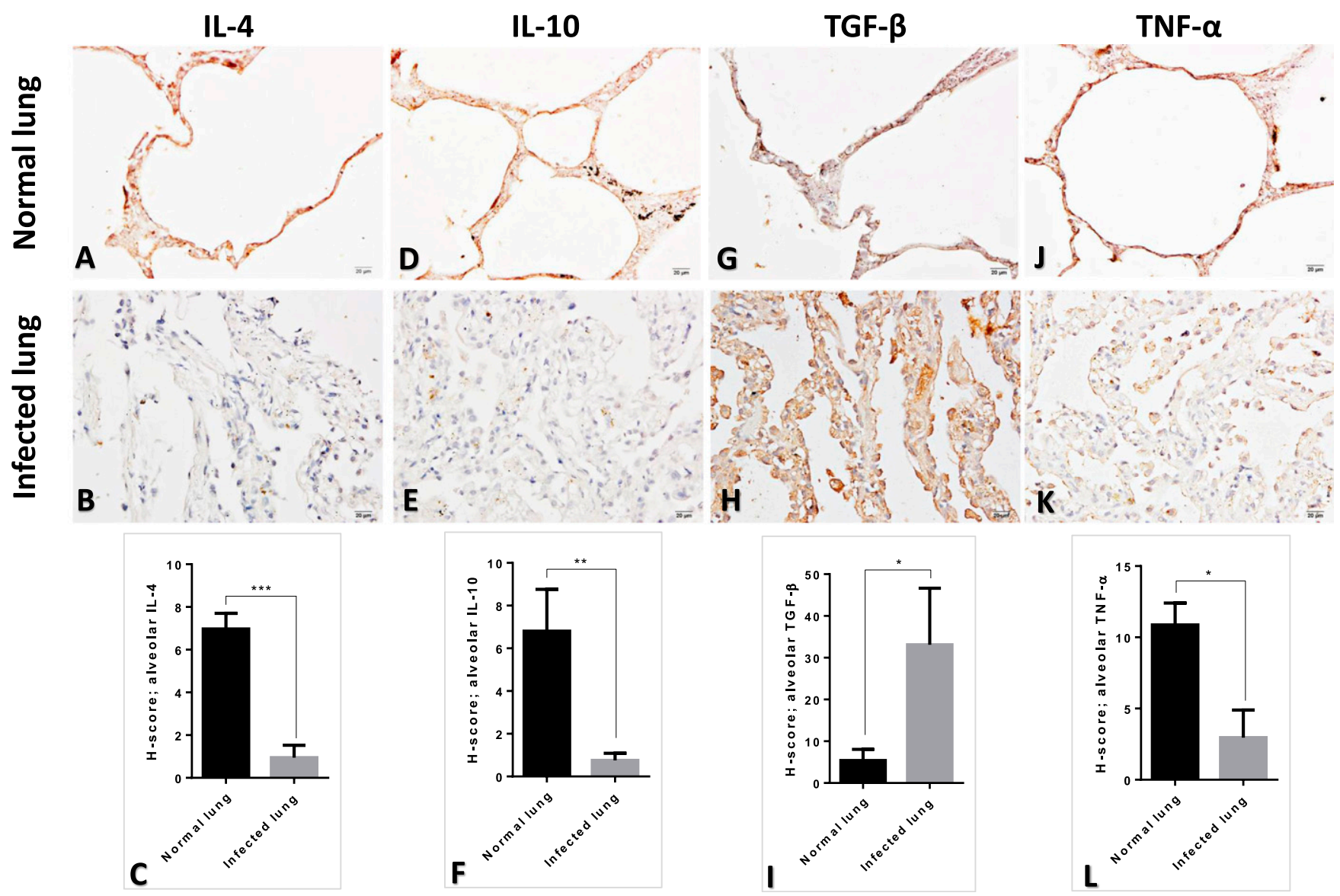

FIGURE 5. Expressions of anti-inflammatory and pro-inflammatory cytokines in the alveolar tissue from the biopsied lung tissue compared with the normal tissue were demonstrated. A-L: Immunohistochemical staining of interleukin (IL)-4, IL-10, transforming growth factor- $\beta$, and tumor necrotic factor- $\alpha(\mathbf{A}-\mathbf{C}, \mathbf{D}-\mathbf{F}, \mathbf{G}-\mathbf{I}$, and $\mathbf{J}-\mathbf{L}$, respectively) scoring with $H$-score $(\mathbf{C}, \mathbf{F}, \mathbf{I}$, and $\mathbf{L})$ between patient lung biopsy $(\mathbf{B}, \mathbf{E}, \mathbf{H}$, and $\mathbf{K})$ and normal lung tissue (A, D, G, and $\mathbf{J})$. This figure appears in color at www.ajtmh.org.

Promthong, Department of Pulmonary and Critical Care Medicine, Maharaj Nakhon Si Thammarat Hospital, Thailand, E-mail: $\mathrm{p}_{-}$ kingpeth@hotmail.com. Paron Dekumyoy, Wallop Pakdee, Kittipong Chaisiri, and Dorn Watthanakulpanich, Department of Helminthology, Faculty of Tropical Medicine, Mahidol University, Bangkok, Thailand, E-mails: paron.dek@mahidol.edu, wallop.pak@mahidol.ac.th, kittipong.chaisiri@gmail.com, and dorn.wat@mahidol.edu. Parnpen Viriyavejakul and Sumate Ampawong, Department of Tropical Pathology, Faculty of Tropical Medicine, Mahidol University, Bangkok, Thailand, E-mails: parnpen.vir@mahidol.ac.th and am_sumate@ hotmail.com.

\section{REFERENCES}

1. Dekumyoy P, Watthanakulpanich D, 2019. Gnathostomiasis. Magill AJ, Hill DR, Solomom T, Ryan ET, eds. Hunter's Tropical Medicine and Emerging Infectious Diseases, 9th edition. London, United Kingdom: Saunders, 853-855.

2. Dekumyoy P, Yoonuan T, Waikagul J, 2012. Gnathostoma Liu D, ed. Molecular Detection of Human Parasitic Pathogens. Boca Raton, FL: CRC Press Taylor \& Francis Group, 563-570.

3. Diaz JH, 2015. Gnathostomiasis: an emerging infection of raw fish consumers in Gnathostoma nematode-endemic and nonendemic countries. J Travel Med 22: 318-324.

4. Pal M, Seid H, Giro B, Wakjira B, Abdo J, 2013. Gnathostomiasis-an emerging nematodal zoonotic disease. Int J Livest Res 3: 12-17.

5. Morishita K, Faust EC, 1925. Two new cases of human creeping disease (gnathostomiasis) in China, with a note on the infection in reservoir hosts in the China area. $J$ Parasitol 11: 158-162.

6. Sharma C, Piyaphanee W, Watthanakulpanich D, 2017. Case report: clinical features of intermittent migratory swelling caused by gnathostomiasis with complete follow-up. Am J Trop Med Hyg 97: 1611-1615.

7. Rusnak JM, Lucey DR, 1993. Clinical gnathostomiasis: case report and review of the English-language literature. Clin Infect Dis 16: 33-50.

8. Katchanov J, Sawanyawisuth K, Chotmongkol V, Nawa Y, 2011. Neurognathostomiasis, a neglected parasitosis of the central nervous system. Emerg Infect Dis 17: 1174.

9. Nawa Y, Yoshikawa M, Sawanyawisuth K, Chotmongkol V, Figueiras SF, Benavides M, Camacho SP, 2017. Ocular gnathostomiasis-update of earlier survey. Am J Trop Med Hyg 97: 1232-1234.

10. Bovornkitti S, Tandhanand S, 1959. A case of spontaneous pneumothorax complicating gnathostomiasis. Dis Chest 35: 328-331.

11. Ampawong S, Chaisri U, Viriyavejakul P, Prapansilp P, Grau GE, Turner GD, Pongponratn E, 2015. A potential role for interleukin-33 and gamma-epithelium sodium channel in the pathogenesis of human malaria associated lung injury. Malar J 14: 389.

12. Maknitikul S, Luplertlop N, Chaisri U, Maneerat $Y$, Ampawong S, 2018. Featured article: immunomodulatory effect of hemozoin on pneumocyte apoptosis via CARD9 pathway, a possibly retarding pulmonary resolution. Exp Biol Med (Maywood) 243: 395-407.

13. Maknitikul S, Luplertlop N, Grau GER, Ampawong S, 2017. Dysregulation of pulmonary endothelial protein $C$ receptor and thrombomodulin in severe falciparum malaria-associated ARDS relevant to hemozoin. PLoS One 12: e0181674.

14. Dekumyoy P, Watthanakulpanich D, Waikagul J, 2014. Helminthnematode: gnathostoma spinigerum. Motarjemi Y, ed. 
Encyclopedia of Food Safety. Waltham, MA: Academic Press, 94-98.

15. Rojekittikhun W, Waikagul J, Chaiyasith T, 2003. Fish as the natural second intermediate host of Gnathostoma spinigerum. Southeast Asian J Trop Med Public Health 33: 63-69.

16. Rojekittikhun W, Pubampen S, Waikagul J, 1998a. Swamp eels (Fluta alba), the genuine second intermediate host of Gnathostoma in Thailand. J Trop Med Parasitol 21: 44-45.

17. Rojekittikhun W, Pubampen S, Waikagul J, 1998b. Seasonal variation in the intensity of Gnathostoma larvae in swamp eels (Fluta alba) sold in a local market of Bangkok. Southeast Asian J Trop Med Public Health 29: 148-153.

18. Daengsvang S, 1980. A Monograph on the Genus Gnathostoma and Gnathostomiasis in Thailand. Tokyo, Japan: Southeast Asian Medical Information Center (SEAMIC), International Medical Foundation of Japan.

19. Prommas C, Daengsvang S, 1934. Nine cases of human gnathostomiasis. Indian Med Gaz 69: 207.

20. Daengsvang S, 1949. Human gnathostomiasis in Siam with reference to the method of prevention. $J$ Parasitol 35: 116-121.

21. Thuraisingam V, AIRP, Sandosham AA, 1969. A presumptive case of gnathostomiasis in Malaysia. Med J Malaya 24: 107-112.

22. Nagler A, Pollack S, Hassoun G, Kerner H, Barzilai D, Lengy J, 1983. Human pleuropulmonary gnathostomiasis: a case report from Israel. Israel J Med Sci 19: 834-837.

23. Kangsadal $P$, Bovornkitti $S, 1960$. A case of gnathostomiasis with spontaneous hydropneumothorax. J Trop Med Hyg 63: 67-70.

24. Nitidandhaprabhas $P$, Hanchansin S, Vongsloesvidhya Y, 1975. A case of expectoration of Gnathostoma spinigerum in Thailand. Am J Trop Med Hyg 24: 547-548.

25. Robert L, 1922. Le Gnathostomose Humaine: Oedéme ambulant siamois dû á Gnathostomum spinigerum (R. Owen, 1836). Bull Soc Pathol Exot 15: 854-860.
26. Prijyanonda B, Pradatsundarasar A, Viranuvatti V, 1955. Pulmonary gnathostomiasis. A case report. Ann Trop Med Parasitol 49: 121-122.

27. Fu XH, Zhou XY, Li X, 1999. Gnathostomiasis in the lung. Chin J Parasitic Dis Contr 12: 142.

28. Nawa Y, Maruyama H, Ogata K, 1997. Current status of gnathostomiasis dorolesi in Miyazaki Prefecture, Japan. Southeast Asian J Trop Med Public Health 28: 11-13.

29. Herman JS, Chiodini PL, 2009. Gnathostomiasis, another emerging imported disease. Clin Microbiol Rev 22: 484-492.

30. Miyazaki I, 1960. On the genus Gnathostoma and human gnathostomiasis, with special reference to Japan. Exp Parasitol 9: 338-370.

31. Cox FE, Liew EY, 1992. Centrefold: T-cell subsets and cytokines in parasitic infections. Parasitol Today 8: 371-374.

32. Hall LR, Mehlotra RK, Higgins AW, Haxhiu MA, Pearlman E, 1998. An essential role for interleukin-5 and eosinophils in helminth-induced airway hyperresponsiveness. Infect Immun 66: 4425-4430.

33. Grainger JRet al., 2010. Helminth secretions induce de novo T cell Foxp3 expression and regulatory function through the TGFbeta pathway. $J$ Exp Med 207: 2331-2341.

34. Benjathummarak S, Kumsiri R, Nuamtanong S, Kalambaheti T, Waikagul J, Viseshakul N, Maneerat Y, 2016. Third-stage Gnathostoma spinigerum larva excretory secretory antigens modulate function of Fc gamma receptor I-mediated monocytes in peripheral blood mononuclear cell culture. Trop Med Health 44: 5.

35. Tsunawaki S, Sporn M, Ding A, Nathan C, 1998. Deactivation of macrophages by transforming growth factor-beta. Nature 334 : 260-262. 